\title{
Strong Bias Effect on Voltage-Driven Torque at Epitaxial Fe-MgO Interface
}

\author{
Shinji Miwa, ${ }^{1,2,}$ Junji Fujimoto, ${ }^{3}$ Philipp Risius, ${ }^{1}$ Kohei Nawaoka, ${ }^{1}$ Minori Goto, ${ }^{1,2}$ and Yoshishige Suzuki ${ }^{1,2}$ \\ ${ }^{1}$ Graduate School of Engineering Science, Osaka University, Toyonaka, Osaka 560-8531, Japan \\ ${ }^{2}$ Center for Spintronics Research Network, Osaka University, Toyonaka, Osaka 560-8531, Japan \\ ${ }^{3}$ Institute for Chemical Research, Kyoto University, Uji, Kyoto 611-0011, Japan \\ (Received 12 February 2017; revised manuscript received 7 June 2017; published 26 July 2017)
}

\begin{abstract}
Torque can be provided to magnetization in nanomagnets directly by electric current and/or voltage. This technique enables electric current (voltage)-to-spin conversion without electromagnetic induction, and has been intensively studied for memory device applications. Among the various kinds of torque, torque induced by spin-orbit splitting has recently been found. However, quantitative understanding of bulkrelated torque and interface-related torque is still lacking because of their identical symmetry for currentin-plane devices. In this paper, we propose that a pure interface-related torque can be characterized by spintorque ferromagnetic resonance with a current-perpendicular-to-plane tunnel junction. Epitaxial $\mathrm{Fe}$ $\mathrm{MgO}-\mathrm{V}$ tunnel junctions are prepared to characterize the interface-related torque at $\mathrm{Fe}-\mathrm{MgO}$. We find that the current-driven torque is negligible, and a significant enhancement of the voltage-driven torque is observed when the $\mathrm{MgO}$ barrier thickness decreases. The maximum torque obtained is as large as $2.8 \times 10^{-5} \mathrm{~J} /\left(\mathrm{Vm}^{2}\right)$, which is comparable to the voltage-controlled magnetic anisotropy of $180 \mathrm{fJ} / \mathrm{Vm}$. The voltage-driven torque shows strong dc-bias-voltage dependence that cannot be explained by conventional voltage-controlled magnetic anisotropy. Tunnel anisotropic magnetoresistance spectroscopy suggests that the torque is correlated to an interface state at the Fe-MgO. This surface-state-sensitive electric modulation of magnetic properties provides new insight into the field of interface magnetism.
\end{abstract}

DOI: 10.1103/PhysRevX.7.031018

\section{INTRODUCTION}

Electric current or voltage provides torque to magnetization in nanomagnets. This enables electric current (voltage)-to-spin conversion without electromagnetic induction and has been intensively studied.

Current-driven spin-transfer torque $[1,2]$ has been studied for memory device applications $[3,4]$. The spin-transfer torque can arise in a current-perpendicular-to-plane (CPP) magnetic tunnel junction following spin accumulation induced by spin injection from a ferromagnetic counter electrode [5-8]. The spin-transfer torque can also arise in current-in-plane (CIP) multilayers. In the CIP multilayers, spin accumulation can be induced by spin-dependent scattering in bulk nonmagnetic metals [9-16] or surface conductive states of topological insulators [17-19], which is termed the spin-Hall or Rashba-Edelstein effect. Spinorbit splitting at a ferromagnet-nonmagnet interface can also induce current-driven torque [11], but the existence of such a torque is still under debate [15]. This is because both the bulk-related and interface-related torques have the same

*miwa@mp.es.osaka-u.ac.jp

Published by the American Physical Society under the terms of the Creative Commons Attribution 4.0 International license. Further distribution of this work must maintain attribution to the author(s) and the published article's title, journal citation, and DOI.
Subject Areas: Spintronics

symmetry in CIP multilayers with some torque measurement such as spin-torque ferromagnetic resonance (FMR) [20,21].

While electric current is needed to generate the aforementioned torque, torque can also be induced by voltage alone. For the voltage-driven torque, voltage-controlled magnetic anisotropy (VCMA) at a ferromagnet-dielectric interface $[22,23]$ can have a significant influence on the experimentally observed torque vector in CPP magnetic tunnel junctions $[24,25]$. However, in such experiments, a ferromagnetic counter electrode inducing tunnel magnetoresistance (TMR) is indispensable for the torque measurement. Therefore, it is difficult to attain a pure voltage-driven torque vector free from spin-transfer torque induced by the counter electrode.

To characterize a pure torque vector originating from a ferromagnet-nonmagnet interface while avoiding the aforementioned issues, we employed a CPP tunnel junction with a single ferromagnetic electrode in the present study. First, the system enables us to characterize the interface-related torque free from the bulk-related one. An electric current flows in a rotational symmetry axis in the CPP tunnel junction; hence, there is no spin accumulation induced by the bulk-spindependent scattering. On the other hand, torque from ferromagnet-dielectric interface can be observed, which is understood as the inverse effect of tunnel anisotropic magnetoresistance (TAMR) [26]. Second, any influence of the spin-injection-induced spin-transfer effect could be 
excluded because of the nonmagnetic counter electrode. Therefore, the system enables us to discuss the interfacerelated torque over a wide current density range. Instead of TMR, spin-torque FMR could be performed using TAMR [27]. Moreover, while spin-torque FMR in a CIP multilayer only provides the torque vector direction $[12,28]$, it is feasible to conduct quantitative characterization of the vector components by employing a CPP tunnel junction $[29,30]$.

We prepared epitaxial Fe-MgO-V tunnel junctions with various $\mathrm{MgO}$ thicknesses, giving a wide range of current density. As a result, we found that the interface-related current-driven torque was negligible, but the voltagedriven torque increased as the $\mathrm{MgO}$ thickness decreased. The maximum voltage-driven torque was as large as $2.8 \times 10^{-5} \mathrm{~J} /\left(\mathrm{Vm}^{2}\right)$. This is comparable to the VCMA at a $\mathrm{Fe}-\mathrm{MgO}$ interface of $180 \mathrm{fJ} / \mathrm{Vm}$, which is larger than any reported VCMA-induced voltage-driven torque [24,25,31-35]. The voltage-driven torque shows nonlinear dc bias voltage dependence, with a maximum around zero dc bias; thus, an electronic state in the vicinity of the Fermi level at a $\mathrm{Fe}-\mathrm{MgO}$ interface is likely to be of importance to generate the large voltage-driven torque. An interface state at $\mathrm{Fe}-\mathrm{MgO}$ was also found in the vicinity of the Fermi level from the TAMR spectroscopy.

\section{EXPERIMENTAL}

Figure 1(a) shows a schematic of the device structure. An epitaxial single crystalline multilayer of $\mathrm{MgO}(001)$ substrate- $\mathrm{MgO}$ buffer $(5 \mathrm{~nm})-\mathrm{V}$ buffer(30 nm)$\mathrm{Fe}(0.74 \mathrm{~nm})-\mathrm{MgO}\left(t_{\mathrm{MgO}}=0.8-1.8 \mathrm{~nm}\right)-\mathrm{V}(3 \mathrm{~nm})-$ $\mathrm{Au}(30 \mathrm{~nm})$ was fabricated by molecular beam epitaxy under ultrahigh vacuum. The $\mathrm{V}$ layer was employed because of its good lattice match with $\mathrm{MgO}$ and because it can induce perpendicular magnetic anisotropy in the $\mathrm{Fe}$ $[36,37]$. Because the counter electrode is nonmagnetic, it is possible to characterize the torque vector independently from the spin-injection-induced spin-transfer torque. During deposition of the $\mathrm{MgO}$ buffer and the $\mathrm{V}$ buffer, substrate temperature was maintained at $150{ }^{\circ} \mathrm{C}$. The $\mathrm{V}$ buffer was post-annealed at $500{ }^{\circ} \mathrm{C}$ for 30 minutes. Other layers were deposited at room temperature. The multilayer was then patterned into 390-nm-diameter tunnel junctions. Figure 1(b) shows reflection high-energy electron diffraction images for the surfaces of the V (001) buffer, Fe (001), and $\mathrm{MgO}(001)$ barrier, where the incident electron beam was parallel to the [100] and [110] azimuths of the $\mathrm{MgO}$ substrate. Sharp streak patterns were observed in all layers, indicating the formation of epitaxial and flat interfaces.

Figure 2(a) shows the device resistance as a function of the $\mathrm{MgO}$ barrier thickness $\left(t_{\mathrm{MgO}}\right)$. The device resistance increased exponentially as the $\mathrm{MgO}$ barrier thickness increased, indicating tunneling conduction. The right axis of Fig. 2(a) shows the resistance-area product (RA), defined as the device resistance multiplied by the junction area. Figure 2(b) shows a typical magnetoresistance (MR) curve (a)

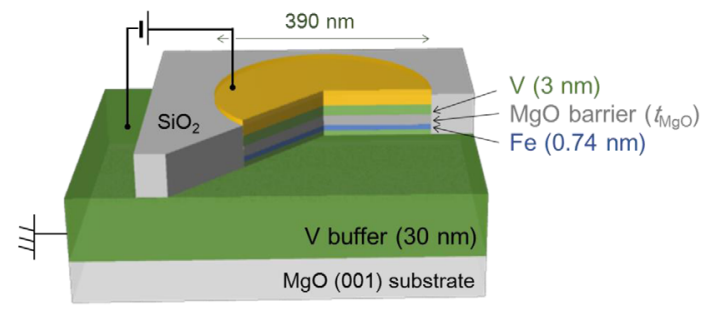

(b)
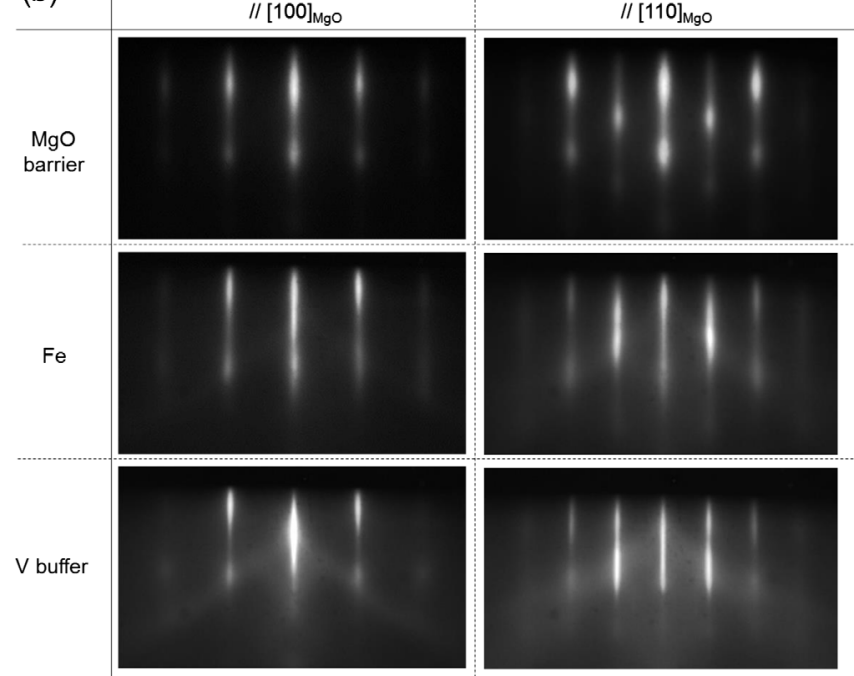

FIG. 1. (a) Schematic illustration of the device structure. (b) In-situ reflection high-energy electron-diffraction images of surfaces of the $\mathrm{MgO}$ barrier, $\mathrm{Fe}$, and $\mathrm{V}$ buffer.

of the device. The results measured from a tunnel junction with $t_{\mathrm{MgO}}=0.94 \mathrm{~nm}$ are shown. When the magnetic-field angle is perpendicular to the film plane $\left(\theta_{H}=90^{\circ}\right)$, the device shows positive magnetoresistance. In an ultrathin Fe layer, shape anisotropy and interface anisotropy at a $\mathrm{Fe}-\mathrm{MgO}$ interface can be comparable [37], and an external magnetic field easily changes its magnetizations from inplane to perpendicular. The MR has the same symmetry as the anisotropic magnetoresistance in ferromagnetic metals [38]. Figure 2(c) shows the variation of the MR ratio, defined as the difference in the saturation resistances between perpendicular and in-plane magnetic fields, as a function of the $\mathrm{MgO}$ barrier thickness. The MR ratio is almost identical when the $\mathrm{MgO}$ barrier thickness increases. Hence, the observed MR in a Fe-MgO- $\mathrm{V}$ tunnel junction is not anisotropic magnetoresistance in the metallic Fe layer but TAMR at the $\mathrm{Fe}-\mathrm{MgO}$ interface. Figure 2(c) shows that the MR ratio decreases for $\mathrm{MgO}$-barrier thicknesses below $0.9 \mathrm{~nm}$. It shows that for $t_{\mathrm{MgO}}<0.9 \mathrm{~nm}$, the resistance of the $\mathrm{MgO}$ barrier does not govern the device resistance, and the resistance of the metal electrode, which has no MR, significantly contributes to the total device resistance.

Figure 3(a) shows a schematic of the experimental setup for torque characterization, in which the spintorque FMR combined with TAMR was employed [27]. 
(a)

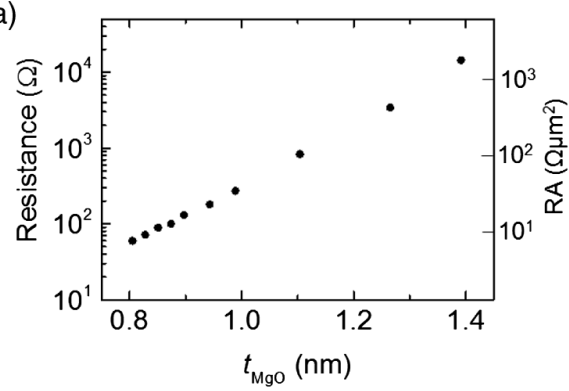

(b)

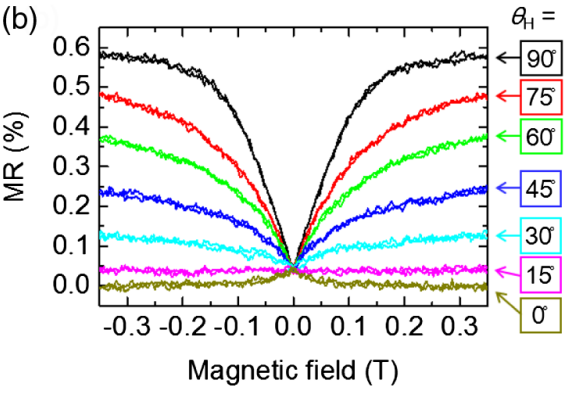

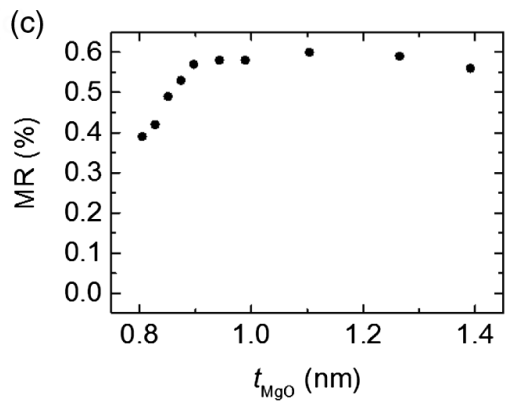

FIG. 2. (a) Device resistance as a function of the $\mathrm{MgO}$ barrier thickness $\left(t_{\mathrm{MgO}}\right)$. (b) Typical TAMR, where $t_{\mathrm{MgO}}$ and resistance are $0.94 \mathrm{~nm}$ and $189 \Omega$, respectively. The magnetic-field angle definition $\left(\theta_{H}\right)$ is depicted in Fig. 3(a). (c) Magnetoresistance ratio as a function of $t_{\mathrm{MgO}}$.

Microwave-frequency voltage was applied through a bias tee to the tunnel junction, and the dc voltage generated in the tunnel junction was measured with a lock-in amplifier. Figure 3(b) shows the definition of the torque vector. The blue arrow represents a unit spin vector of the Fe. The torque vector components $\left(\beta_{/ /}, \beta_{\perp}\right)$ can be expressed as the following modified Landau-Lifshitz-Gilbert equation:

$$
\begin{aligned}
\frac{d \mathbf{s}}{d t}= & \gamma_{0} \mathbf{s} \times \mathbf{H}_{\mathrm{eff}}-\alpha \mathbf{s} \times \frac{d \mathbf{s}}{d t}-\frac{A}{S}\left(\beta_{/ /} \mathbf{s} \times(\mathbf{z} \times \mathbf{s})\right. \\
& \left.+\beta_{\perp}(\mathbf{z} \times \mathbf{s})\right) \sqrt{\eta} V_{\mathrm{rf}},
\end{aligned}
$$

where $\mathbf{s}$ and $\mathbf{z}$ are the unit vectors of $\mathrm{Fe}$ and the out-of-plane direction, respectively. $\mathbf{H}_{\text {eff }}$ is the effective magnetic field applied to the $\mathrm{Fe} ; \gamma_{0}(<0)$; and $\alpha, S$, and $A$ are the gyromagnetic ratio, Gilbert damping constant, and total spin-angular momentum in the Fe layer and area of the tunnel junction, respectively. Here, $\eta$ is the transmission efficiency of voltage due to the impedance mismatch between the tunnel junction and the transmission line, and $V_{\mathrm{rf}}$ is the amplitude of the voltage in the transmission line from the microwave generator. Hence, the amplitude of $\sqrt{\eta} V_{\text {rf }}$ is the applied microwave-frequency voltage in the tunnel junction. The definition and experimental method to obtain $\eta$ are provided in our previous paper [24]. In a precise sense, $\gamma_{0}$ should be a tensor. However, during FMR measurements, the magnetization angle did not change significantly. Hence, we treated it as a constant. Note that $\mathbf{z}$ is the rotational symmetry axis if we do not take into account the fourfold crystal structure in the system. Therefore, $\mathbf{z}$ should be the reference vector in the system, and the coordinates of $\mathbf{s} \times(\mathbf{z} \times \mathbf{s})$ and $\mathbf{z} \times \mathbf{s}$ are employed to express the torque vector $\left(\beta_{/ /}, \beta_{\perp}\right)$. Although there can be an in-plane symmetry breaking axis due to the crystal structure of $\mathrm{Fe}$, the $\mathbf{z}$ reference axis is a standard and most suitable choice because we apply the current or voltage in the $\mathbf{z}$ direction. In all of the experiments in this study, the direction of in-plane projection of $\mathbf{s}$ is set to $\mathbf{x}$, which corresponds to [100] azimuth of the Fe. Figure 3(c) shows typical spin-torque FMR spectra of a tunnel junction with $t_{\mathrm{MgO}}=0.94 \mathrm{~nm}$ and microwave power of $0.1 \mu \mathrm{W}$. A magnetic field tilted $45^{\circ}$ from the film plane was applied to maximize the dc voltage signals. To characterize the torque vector, the obtained spectra were fit by the following equation $[20,21]$ :

$$
\begin{aligned}
V_{\mathrm{L}} & \frac{(\Delta H)^{2}}{\left(H-H_{\mathrm{FMR}}\right)^{2}+(\Delta H)^{2}} \\
& +V_{\mathrm{AL}} \frac{(\Delta H)^{2}}{\left(H-H_{\mathrm{FMR}}\right)^{2}+(\Delta H)^{2}} \frac{H-H_{\mathrm{FMR}}}{\Delta H}+a H+b .
\end{aligned}
$$

Equation 2 consists of the linear combination of the Lorentzian function, the first term with the fitting parameter $V_{L}$ and the anti-Lorentzian function, and the second term with the fitting parameter $V_{\mathrm{AL}}$. Here, $H$ is an external magnetic field, and $V_{L}, V_{\mathrm{AL}}$, resonant field $\left(H_{\mathrm{FMR}}\right)$, linewidth $(\Delta H)$, and linear background $(a, b)$ are taken as fitting parameters. From the fitting parameters of $V_{L}$ and $V_{\mathrm{AL}}$, torque vector components of $\beta_{/ /}$and $\beta_{\perp}$ can be obtained using the following equation:

$$
\begin{aligned}
V_{\mathrm{L}(\mathrm{AL})}= & -\frac{1}{2} \sin \theta \sin 2 \theta \frac{R(\theta)\left(R_{\theta=0^{\circ}}-R_{\theta=90^{\circ}}\right)}{R_{\theta=0^{\circ}} R_{\theta=90^{\circ}}} \\
& \times \frac{\beta_{/ /(\perp)}}{2 \pi \gamma_{0} \Delta H} \eta\left(V_{\mathrm{RF}}\right)^{2} .
\end{aligned}
$$

To derive Eq. (3), we employed the analysis provided in Ref. [24] and angle dependence of the TAMR, $R^{-1}(\theta)=$ $\left(R_{\theta=90^{\circ}}+R_{\theta=0^{\circ}}{ }^{-1}\right) / 2+\left(R_{\theta=90^{\circ}}-R_{\theta=0^{\circ}}{ }^{-1}\right) / 2 \times \cos 2 \theta$. Here, $R(\theta)$ is the device resistance when the spin-vector direction defined in Fig. 3(b) is $\theta$. From the angle dependence of the TAMR, we can deduce the values of $\theta$ in the torque analysis.

\section{RESULTS AND DISCUSSION}

Figure 4(a) shows torque vector components in $\mathrm{Fe}-\mathrm{MgO}-\mathrm{V}$ tunnel junctions, which were obtained by spin-torque FMR spectra and Eqs. (2) and (3). Black 


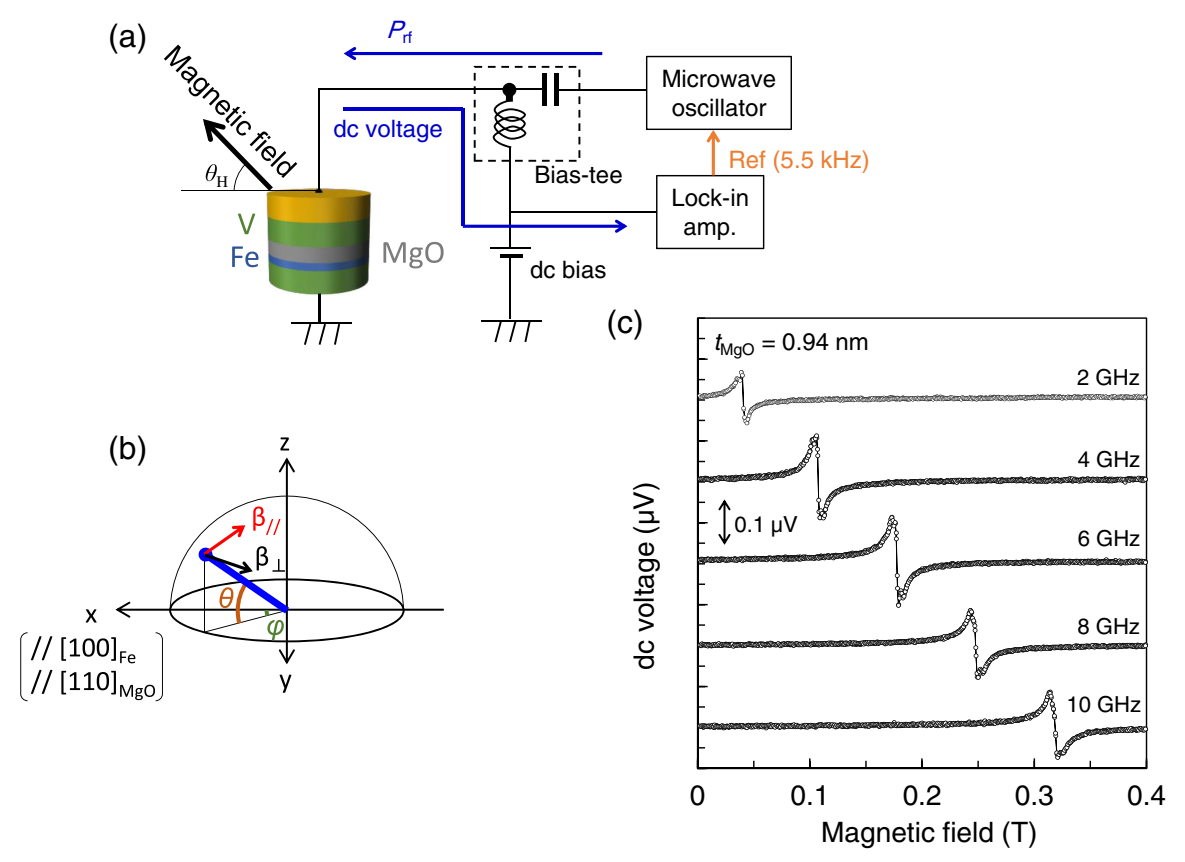

FIG. 3. (a) Schematic illustration of the measurement setup. (b) Definition of perpendicular $\left(\beta_{\perp}\right)$ and in-plane $\left(\beta_{/ /}\right)$torque. (c) Typical spectra for spin-torque FMR. The magnetic-field direction is $\theta_{H}=45^{\circ}$, and the input power $\left(P_{\mathrm{rf}}\right)$ is $0.1 \mu \mathrm{W}$.

and red circles represent perpendicular and in-plane components, respectively. The measurements were conducted under a magnetic-field angle of $\theta_{H}=45^{\circ}$, and input microwave power and frequency were set to $P_{\mathrm{rf}}=$ $0.1 \mu \mathrm{W}$ and $5 \mathrm{GHz}$, respectively. First, a significant inplane component was not observed in every device, and the perpendicular component was dominant. The theory predicted that the current-driven in-plane torque could be dominant because of interfacial spin-orbit splitting [26], but this is inconsistent with our observation. This does not mean that there are some problems with the theoretical estimation of the in-plane torque in Ref. [26]. The inconsistency might be because the simple model employed in Ref. [26] did not include the physics that can induce the large perpendicular torque in our experiment. Second, the perpendicular torque is voltage driven. The variation of the perpendicular torque in Fig. 4(a) is at most twice as large, whereas the current density of the tunnel junction with $t_{\mathrm{MgO}}=0.8 \mathrm{~nm}$ is 140 times larger than that with $t_{\mathrm{MgO}}=$ $1.4 \mathrm{~nm}$. Therefore, the perpendicular component is not current driven but voltage driven. Note that the current-induced magnetic field cannot be the origin of the voltage-driven perpendicular torque. Any influence of the spin-Hall effect from the $\mathrm{V}$ underlayer is also excluded in the same manner.

We have also characterized the in-plane magnetization direction $(\varphi)$ dependence of the torque, but significant change was not observed, which also supports our premise that the current-induced magnetic field and the spin-Hall effect are not the origin of the torque. The analysis above shows that the voltage-driven perpendicular torque originates from the $\mathrm{Fe}-\mathrm{MgO}$ interface. The dashed curve in
Fig. 4(a) shows that the maximum torque was about $2.8 \times 10^{-5} \mathrm{~J} /\left(\mathrm{Vm}^{2}\right)$, which is the same as the voltagedriven torque induced by the large VCMA of $180 \mathrm{fJ} / \mathrm{Vm}$ at the $\mathrm{Fe}-\mathrm{MgO}$ interface. To the best of our knowledge, this is larger than any previously reported VCMA-induced voltage-driven torque [24,25,31-35].

Figure 4(b) shows the input power dependence of peak-to-peak dc voltage in the spin-torque FMR spectra of devices with $t_{\mathrm{MgO}}=0.94 \mathrm{~nm}$. The microwave frequency was $5 \mathrm{GHz}$. A linear relation between the peak-to-peak voltage and the input power is only confirmed in the small input power region, namely, less than $10^{-4} \mathrm{~mW}$. This indicates that large spin precession is excited by only a small input power, which is consistent with the large torque vector components obtained in Fig. 4(a). More quantitatively, the magnetization precession angle $\left(1.5^{\circ}\right.$ at $P_{\mathrm{rf}}=10^{-4} \mathrm{~mW}$ ) is of the same order as that of the highly sensitive spin-torque diode device with low resistance-area product $\left(2.5 \Omega \mu \mathrm{m}^{2}, 3.6^{\circ}\right.$ at $\left.P_{\mathrm{rf}}=10^{-4} \mathrm{~mW}\right)$ [8].

To characterize the VCMA at the Fe-MgO interface in the device, the dc-bias-voltage dependence of the resonant field was characterized as shown in Fig. 4(c). Microwave frequency and power were $5 \mathrm{GHz}$ and $0.1 \mu \mathrm{W}$, respectively. Figure 4(c) shows that the resonant field changes almost linearly as a function of the dc bias voltage. The resonant field change corresponds to a VCMA of about $20 \mathrm{fJ} / \mathrm{Vm}$. Note that the VCMA of $20 \mathrm{fJ} / \mathrm{Vm}$ is too small to reproduce the observed large perpendicular torque component in Fig. 4(a).

Figure 4(d) shows a dc-bias-voltage dependence of the perpendicular torque component. The torque was 
(a)

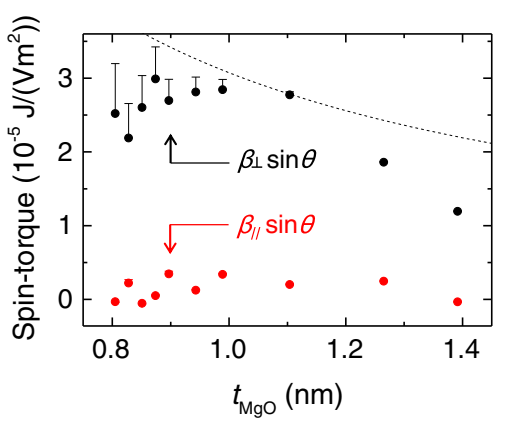

(b)

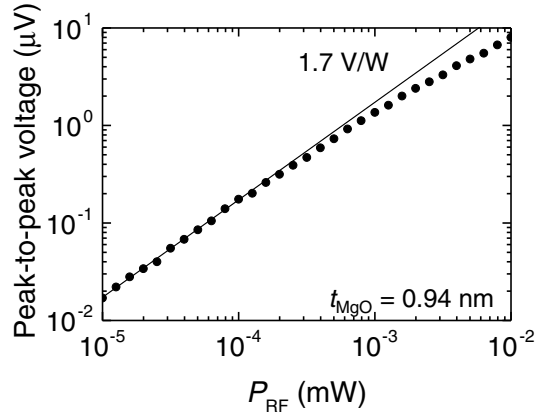

(c)

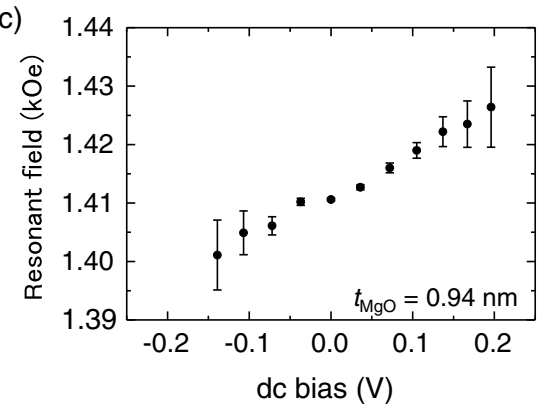

(d)

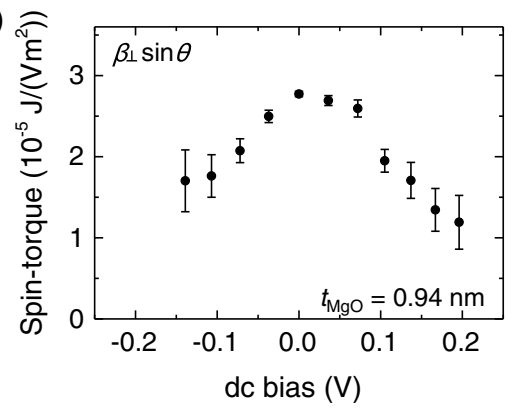

FIG. 4. (a) Torque vector components. Black and red circles represent perpendicular $\left(\beta_{\perp}\right)$ and in-plane $\left(\beta_{/ /}\right)$torque, respectively. The dashed curve shows the theoretical line of the torque induced by the voltage-controlled magnetic anisotropy of $180 \mathrm{fJ} / \mathrm{Vm}$. (b) Input power dependence of peak-to-peak dc voltage in the spin-torque FMR spectra. (c) The dc-bias-voltage dependence of the resonant field. (d) The dc-bias-voltage dependence of the perpendicular torque. All data were obtained with a magnetic-field angle of $\theta_{H}=45^{\circ}$ and an input microwave frequency of $5 \mathrm{GHz}$.

characterized by Eq. (3), where dc-bias-voltage dependence of the MR ratio is included. The torque shows nonlinear dc-bias-voltage dependence and takes its maximum around zero dc bias. The dependence is unique because the reported VCMA at a Fe-MgO interface is linear [24,25,31-33]; hence, VCMA-induced voltagedriven torque should be independent of the dc bias.

From the discussion above, the Fe-MgO-V tunnel junction shows large voltage-driven perpendicular torque. The torque originates from the $\mathrm{Fe}-\mathrm{MgO}$ interface; however, it cannot be explained by conventional VCMA at the Fe$\mathrm{MgO}$ interface. Namely, the spin torque is equivalent to a dynamic VCMA of $180 \mathrm{fJ} / \mathrm{Vm}$; however, the Fe-MgO interface shows a static VCMA of only $20 \mathrm{fJ} / \mathrm{Vm}$. In the previous studies of $\mathrm{Fe}(\mathrm{Co})-\mathrm{MgO}$ interfaces, a static VCMA of 20-30 fJ/ Vm was reported [24,37,39], which is consistent with the present study. In addition, dynamic VCMA was observed, as the voltage-driven torque was basically identical to the static VCMA $[24,25,32,33]$. Contrary to this, the dynamic VCMA is several times larger than that of the static VCMA in the present study.

Figure 4(d) shows that electronic states at the $\mathrm{Fe}-\mathrm{MgO}$ interface around the Fermi level are likely to have a strong influence on the unique torque property. Hence, to characterize the electronic state of the $\mathrm{Fe}-\mathrm{MgO}$ interface, the dc-bias-voltage dependence of the TAMR was characterized. For this purpose, a device with $t_{\mathrm{MgO}}=1.77 \mathrm{~nm}$, with $10-\mu \mathrm{m}^{2}$-sized junctions, was fabricated. The magnetoresistance under $1.5 \mathrm{~T}$ at various magnetic field angles was measured using the dc two-terminal method, and it is displayed in Fig. 5(a) as black circles. The data in Fig. 5(a) can be fitted using the following equations $[40,41]$ :

$$
\begin{gathered}
R(\theta)^{-1}=\sum_{n=0}^{4} G_{2 n \theta} \cos 2 n \theta, \\
\frac{\cos \theta}{\sin \theta}=\frac{H \cos \theta_{\mathrm{H}}-H_{d} \cos \theta}{H \sin \theta_{\mathrm{H}}} .
\end{gathered}
$$

Here, $H, \theta$, and $\theta_{H}$ are, respectively, the magnetic field strength, magnetization angle, and magnetic field angle defined in Fig. 3(a). Note that $H_{d}$ is the saturation magnetic field in the perpendicular direction $\left(\theta_{H}=90^{\circ}\right)$, and $G_{2 n \theta}$ are fitting coefficients. The red curves in Fig. 5(a) are the fit results. Fit coefficients of twofold $\left(G_{2 \theta} / G_{0}\right)$ and fourfold $\left(G_{4 \theta} / G_{0}\right)$ components are depicted in Figs. 5(b) and 5(c). The error bars in Figs. 5(b) and 5(c) are the results when $H_{d}$ varies from 0.4 to $0.7 \mathrm{~T}$.

The TAMR is correlated with electronic states at the $\mathrm{Fe}-\mathrm{MgO}$ interface [40-42]. The origin of the twofold component in the TAMR is similar to the AMR. The twofold component can appear if one symmetry axis exists: that is, electric current direction and spin-dependent scattering due to the spin-orbit interaction at the $\mathrm{Fe}-\mathrm{MgO}$ interface. The fourfold component cannot be explained in the same manner. It should be correlated with the crystal 
(a)

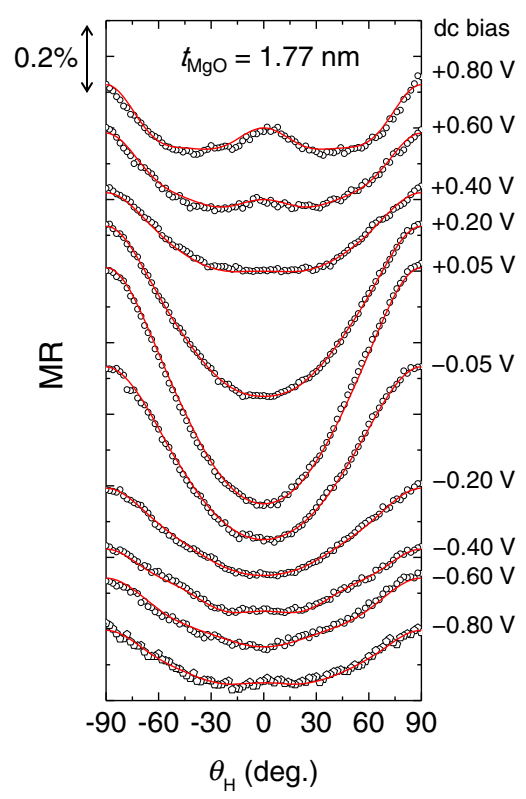

(b)

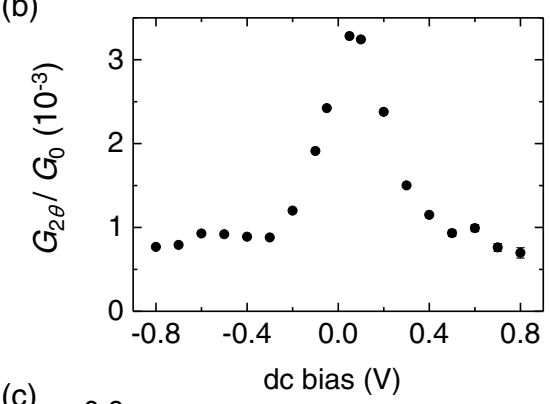

(c)

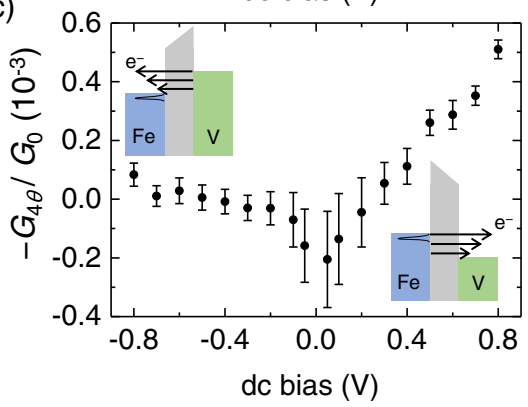

FIG. 5. (a) The dc-bias-voltage dependence of TAMR. Black circles and red curves are experimental data and fitting curves using Eq. 4, respectively. (b) Twofold component of the TAMR. (c) Fourfold component of the TAMR.

structure of $\mathrm{Fe}$ and/or $\mathrm{MgO}$ that has fourfold symmetry. As shown in the previous study [40], the fourfold component in the TAMR indicates the existence of an interface state at the $\mathrm{Fe}-\mathrm{MgO}$ interface. Figure 5(c) shows that the fourfold component is not significant under negative dc bias and that it is only significant under positive bias. This indicates that an interface state at the $\mathrm{Fe}-\mathrm{MgO}$ interface exists around the Fermi level of the Fe as schematically shown in the inset of Fig. 5(c). An interface resonance state with a similar energy level at the $\mathrm{Fe}-\mathrm{MgO}$ interface is also reported in previous studies [43,44]. Interestingly, the torque component in Fig. 4(d) takes its maximum when the energy level is almost identical to the interface state characterized by the angular dependence of the TAMR as shown in Fig. 5.

As discussed, the Fe-MgO-V tunnel junction in the present study shows unique properties as compared with previous studies $[24,37,39]$. Namely, the dynamic VCMA that induces voltage-driven perpendicular torque is several times larger than the static VCMA. Finally, we would like to discuss unique points in the device structure and possible origins of the large voltage-driven torque.

The $\mathrm{V}$ buffer layer is worth discussing because it is a unique feature of the device structure, and $\mathrm{V}$ can form an alloy with $\mathrm{Fe}$. In addition, because the lattice constants of Fe, $\mathrm{V}$, and $\mathrm{MgO}$ are 0.286, 0.303, and $0.298 \mathrm{~nm}$, respectively, the in-plane lattice constant of the Fe can be expanded. It is reported that the underlayer material can change the VCMA coefficient and its dc-bias-voltage dependence at the $\mathrm{Fe}(\mathrm{CoB})-\mathrm{MgO}$ interface [36,45-49], which can be induced by alloying with the underlayer materials [47] and/or lattice distortion [48,49]. However, in all the studies mentioned above, large modified VCMA was obtained with post-annealing. Contrary to this, postannealing was not conducted in the $\mathrm{Fe}-\mathrm{MgO}$ tunnel junction in the present study. In addition, we have also conducted similar measurements with the same sample post-annealed at $250{ }^{\circ} \mathrm{C}$ for 30 minutes, and we found that the magnitude of the torque was almost identical to asdeposited devices, but the perpendicular magnetic anisotropy and ferromagnetic resonance linewidth of the Fe slightly increased. Therefore, it should be noted that the existence of the large torque is robust to the sample fabrication process. The only unique point in our sample is the small $\mathrm{MgO}$ barrier thickness $(<1.1 \mathrm{~nm})$. All of the previous studies concerning VCMA in magnetic tunnel junctions employed thicker $\mathrm{MgO}$ barriers than the present study.

The influence of electrochemical reactions [50-53] and/ or charge traps [36] should be discussed as a possible origin of the large torque. However, in such cases, dynamic VCMA inducing torque should be smaller than static VCMA. This is because electrochemical reactions and/or charge trap inducing static VCMA have limited operation speed owing to thermal activation processes. Moreover, it is reported that there is no voltage-driven electrochemical reaction that substantially changes a valence state of $d$ orbitals [54-56].

To explain the observed phenomena in a different way, they occur only when very high frequency $(>\mathrm{GHz})$ or small (for instance, $\sqrt{\eta} V_{\mathrm{rf}}$ under $P_{\mathrm{rf}}=10^{-4} \mathrm{~mW}$ is $3.2 \mathrm{mV}$ ) external voltage is applied; thus, a large voltage-driven perpendicular torque was induced. Hence, if the $\mathrm{MgO}$ barrier in the tunnel junctions has some localized state [57] and its capacitance shows dc-bias-voltage dependence, then 
the nonlinearity in the bias dependence of the torque can be explained. In either case, nonlinear dc-bias dependence of the voltage-driven torque in Fig. 4(d) demonstrates the importance of the surface state at the $\mathrm{Fe}-\mathrm{MgO}$ interface. Interestingly, the voltage-driven torque takes its maximum around the interface state at the $\mathrm{Fe}-\mathrm{MgO}$ interface as shown in Fig. 5. For further discussion, a theoretical approach including a first-principles study would be indispensable.

\section{CONCLUSION}

To conclude, the torque vector at an epitaxial $\mathrm{Fe}-\mathrm{MgO}$ interface was characterized by spin-torque FMR in a CPP $\mathrm{Fe}-\mathrm{MgO}-\mathrm{V}$ tunnel junction. The current-driven torque, which can be induced by an interfacial spin-orbit splitting at $\mathrm{Fe}-\mathrm{MgO}$, was negligible; however, the voltage-driven perpendicular torque component increases as the $\mathrm{MgO}$ barrier thickness decreases. The obtained maximum torque is as large as $2.8 \times 10^{-5} \mathrm{~J} /\left(\mathrm{Vm}^{2}\right)$ for a thin $\mathrm{MgO}$ barrier $(<1.1 \mathrm{~nm})$. The perpendicular torque is comparable to the VCMA at an Fe-MgO interface of $180 \mathrm{fJ} / \mathrm{Vm}$. The voltage-driven torque component shows nonlinear dcbias-voltage dependence, and it takes its maximum when the energy level is in the vicinity of the Fermi level, where the interface state at the Fe-MgO interface is also located. This surface-state-sensitive electric modulation of magnetic properties provides new insight in the field of interface magnetism.

\section{ACKNOWLEDGMENTS}

We thank E. Tamura and Y. Niimi of Osaka University, Y. Shiota of AIST, and H. Kohno of Nagoya University for fruitful discussions. This work was supported by the Grant-in-Aid for Scientific Research on Innovative Area, "Nano Spin Conversion Science" (Grant No. 26103002), JSPS KAKENHI (Grants No. JP15H05420 and No. JP15H05702), The ImPACT Program, and Iketani Science and Technology Foundation, Japan.

[1] L. Berger, Emission of Spin Waves by a Magnetic Multilayer Traversed by a Current, Phys. Rev. B 54, 9353 (1996).

[2] J. C. Slonczewski, Current-Driven Excitation of Magnetic Multilayers, J. Magn. Magn. Mater. 159, L1 (1996).

[3] K. Ando, S. Fujita, J. Ito, S. Yuasa, Y. Suzuki, Y. Nakatani, T. Miyazaki, and H. Yoda, Spin-Transfer Torque Magnetoresistive Random-Access Memory Technologies for Normally off Computing (Invited), J. Appl. Phys. 115, 172607 (2014).

[4] N. Locatelli, V. Cros, and J. Grollier, Spin-Torque Building Blocks, Nat. Mater. 13, 11 (2014).

[5] J. A. Katine, F. J. Albert, R. A. Buhrman, E. B. Myers, and D. C. Ralph, Current-Driven Magnetization Reversal and Spin-Wave Excitations in $\mathrm{Co} / \mathrm{Cu} / \mathrm{Co}$ Pillars, Phys. Rev. Lett. 84, 3149 (2000).
[6] J.Z. Sun, T. S. Kuan, J. A. Katine, and R. H. Koch, Spin Angular Momentum Transfer in a Current-Perpendicular Spin-Valve Nanomagnet, Proc. SPIE Int. Soc. Opt. Eng. 5359, 445 (2004).

[7] H. Tomita, S. Miwa, T. Nozaki, S. Yamashita, T. Nagase, K. Nishiyama, E. Kitagawa, M. Yoshikawa, T. Daibou, M. Nagamine, T. Kishi, S. Ikegawa, N. Shimomura, H. Yoda, and Y. Suzuki, Unified Understanding of Both Thermally Assisted and Precessional Spin-Transfer Switching in Perpendicularly Magnetized Giant Magnetoresistive Nanopillars, Appl. Phys. Lett. 102, 042409 (2013).

[8] S. Miwa, S. Ishibashi, H. Tomita, T. Nozaki, E. Tamura, K. Ando, N. Mizuochi, T. Saruya, H. Kubota, K. Yakushiji et al., Highly Sensitive Nanoscale Spin-Torque Diode, Nat. Mater. 13, 50 (2014).

[9] T. Kimura, Y. Otani, T. Sato, S. Takahashi, and S. Maekawa, Room-Temperature Reversible Spin Hall Effect, Phys. Rev. Lett. 98, 156601 (2007).

[10] K. Ando, S. Takahashi, K. Harii, K. Sasage, J. Ieda, S. Maekawa, and E. Saitoh, Electric Manipulation of Spin Relaxation Using the Spin Hall Effect, Phys. Rev. Lett. 101, 036601 (2008).

[11] I. M. Miron, K. Garello, G. Gaudin, P.-J. Zermatten, M. V. Costache, S. Auffret, S. Bandiera, B. Rodmacq, A. Schuhl, and P. Gambardella, Perpendicular Switching of a Single Ferromagnetic Layer Induced by In-Plane Current Injection, Nature (London) 476, 189 (2011).

[12] L. Liu, T. Moriyama, D. C. Ralph, and R. A. Buhrman, Spin-Torque Ferromagnetic Resonance Induced by the Spin Hall Effect, Phys. Rev. Lett. 106, 036601 (2011).

[13] L. Liu, C.-F. Pai, Y. Li, H. W. Tseng, D. C. Ralph, and R. A. Buhrman, Spin-Torque Switching with the Giant Spin Hall Effect of Tantalum, Science 336, 555 (2012).

[14] Y. Niimi, Y. Kawanishi, D. H. Wei, C. Deranlot, H. X. Yang, M. Chshiev, T. Valet, A. Fert, and Y. Otani, Giant Spin Hall Effect Induced by Skew Scattering from Bismuth Impurities inside Thin Film CuBi Alloys, Phys. Rev. Lett. 109, 156602 (2012).

[15] J. Kim, J. Sinha, M. Hayashi, M. Yamanouchi, S. Fukami, T. Suzuki, S. Mitani, and H. Ohno, Layer Thickness Dependence of the Current-Induced Effective Field Vector in $\mathrm{Ta}|\mathrm{CoFeB}|$, Nat. Mater. 12, 240 (2013).

[16] S. Emori, U. Bauer, S.-M. Ahn, E. Martinez, and G. S. D. Beach, Current-Driven Dynamics of Chiral Ferromagnetic Domain Walls, Nat. Mater. 12, 611 (2013).

[17] A. R. Mellnik, J. S. Lee, A. Richardella, J. L. Grab, P. J. Mintn, M. H. Fischer, A. Vaezi, A. Manchon, E.-A. Kim, N. Samarth, and D. C. Ralph, Spin-Transfer Torque Generated by a Topological Insulator, Nature (London) 511, 449 (2014).

[18] Y. Fan, P. Upadhyaya, X. Kou, M. Lang, S. Takei, Z. Wang, J. Tang, L. He, L.-T. Chang, M. Montazeri et al., Magnetization Switching through Giant Spin-Orbit Torque in a Magnetically Doped Topological Insulator Heterostructure, Nat. Mater. 13, 699 (2014).

[19] K. Kondou, R. Yoshimi, A. Tsukazaki, Y. Fukuma, J. Matsuno, K. S. Takahashi, M. Kawasaki, Y. Tokura, and Y. Otani, Fermi-Level-Dependent Charge-to-Spin Current Conversion by Dirac Surface States of Topological Insulators, Nat. Phys. 12, 1027 (2016). 
[20] A. A. Tulapurkar, Y. Suzuki, A. Fukushima, H. Kubota, H. Maehara, K. Tsunekawa, D. D. Djayaprawira, N. Watanabe, and S. Yuasa, Spin-Torque Diode Effect in Magnetic Tunnel Junctions, Nature (London) 438, 339 (2005).

[21] J. C. Sankey, P. M. Braganca, A. G. F. Garcia, I. N. Krivorotov, R. A. Buhrman, and D.C. Ralph, SpinTransfer-Driven Ferromagnetic Resonance of Individual Nanomagnets, Phys. Rev. Lett. 96, 227601 (2006).

[22] M. Weisheit, S. Fähler, A. Marty, Y. Souche, C. Poinsignon, and D. Givord, Electric Field-Induced Modification of Magnetism in Thin-Film Ferromagnets, Science 315, 349 (2007).

[23] T. Maruyama, Y. Shiota, T. Nozaki, K. Ohta, N. Toda, M. Mizuguchi, A. Tulapurkar, T. Shinjo, M. Shiraishi, S. Mizukami, Y. Ando, and Y. Suzuki, Large Voltage-Induced Magnetic Anisotropy Change in a Few Atomic Layers of Iron, Nat. Nanotechnol. 4, 158 (2009).

[24] T. Nozaki, Y. Shiota, S. Miwa, S. Murakami, F. Bonell, S. Ishibashi, H. Kubota, K. Yakushiji, T. Saruya, A. Fukushima, S. Yuasa, T. Shinjo, and Y. Suzuki, ElectricField-Induced Ferromagnetic Resonance Excitation in an Ultrathin Ferromagnetic Metal Layer, Nat. Phys. 8, 491 (2012).

[25] J. Zhu, J. A. Katine, G. E. Rowlands, Y.-J. Chen, Z. Duan, J. G. Alzate, P. Upadhyaya, J. Langer, P. K. Amiri, K. L. Wang, and I. N. Krivorotov, Voltage-Induced Ferromagnetic Resonance in Magnetic Tunnel Junctions, Phys. Rev. Lett. 108, 197203 (2012).

[26] A. Manchon, Interfacial Spin-Orbit Splitting and CurrentDriven Spin Torque in Anisotropic Tunnel Junctions, Phys. Rev. B 83, 172403 (2011).

[27] S. Hatanaka, S. Miwa, K. Matsuda, K. Nawaoka, K. Tanaka, H. Morishita, M. Goto, N. Mizuochi, T. Shinjo, and Y. Suzuki, Tunnel Anisotropic Magnetoresistance in $\mathrm{CoFeB}|\mathrm{MgO}| \mathrm{Ta}$ Junctions, Appl. Phys. Lett. 107, 082407 (2015).

[28] K. Kondou, H. Sukegawa, S. Kasai, S. Mitani, Y. Niimi, and Y. Otani, Influence of Inverse Spin Hall Effect in SpinTorque Ferromagnetic Resonance Measurements, Appl. Phys. Express 9, 023002 (2016).

[29] H. Kubota, A. Fukushima, K. Yakushiji, T. Nagahama, S. Yuasa, K. Ando, H. Maehara, Y. Nagamine, K. Tsunekawa, D. D. Djayaprawira, N. Watanabe, and Y. Suzuki, Quantitative Measurement of Voltage Dependence of SpinTransfer Torque in MgO-Based Magnetic Tunnel Junctions, Nat. Phys. 4, 37 (2008).

[30] J. C. Sankey, Y.-T. Cui, J. Z. Sun, J. C. Slonczewski, R. A. Buhrman, and D.C. Ralph, Measurement of the SpinTransfer-Torque Vector in Magnetic Tunnel Junctions, Nat. Phys. 4, 67 (2008).

[31] T. Nozaki, H. Arai, K. Yakushiji, S. Tamaru, H. Kubota, H. Imamura, A. Fukushima, and S. Yuasa, Magnetization Switching Assisted by High-Frequency-Voltage-Induced Ferromagnetic Resonance, Appl. Phys. Express 7, 073002 (2014).

[32] Y. Shiota, S. Miwa, S. Tamaru, T. Nozaki, H. Kubota, A. Fukushima, Y. Suzuki, and S. Yuasa, High-Output Microwave Detector Using Voltage-Induced Ferromagnetic Resonance, Appl. Phys. Lett. 105, 192408 (2014).
[33] Y. Shiota, S. Miwa, S. Tamaru, T. Nozaki, H. Kubota, A. Fukushima, Y. Suzuki, and S. Yuasa, Field Angle Dependence of Voltage-Induced Ferromagnetic Resonance under dc Bias Voltage, J. Magn. Magn. Mater. 400, 159 (2016).

[34] Y. Shiota, T. Nozaki, S. Tamaru, K. Yakushiji, H. Kubota, A. Fukushima, S. Yuasa, and Y. Suzuki, Evaluation of Write Error Rate for Voltage-Driven Dynamic Magnetization Switching in Magnetic Tunnel Junctions with Perpendicular Magnetization, Appl. Phys. Express 9, 013001 (2016).

[35] C. Grezes, F. Ebrahimi, J. G. Alzate, X. Cai, J. A. Katine, J. Langer, B. Ocker, P. K. Amiri, and K. L. Wang, Ultra-Low Switching Energy and Scaling in Electric-Field-Controlled Nanoscale Magnetic Tunnel Junctions with High ResistanceArea Product, Appl. Phys. Lett. 108, 012403 (2016).

[36] A. Rajanikanth, T. Hauet, F. Montaigne, S. Mangin, and S. Andrieu, Magnetic Anisotropy Modified by Electric Field in $\mathrm{V} / \mathrm{Fe} / \mathrm{MgO}(001) / \mathrm{Fe}$ Epitaxial Magnetic Tunnel Junction, Appl. Phys. Lett. 103, 062402 (2013).

[37] K. Tanaka, S. Miwa, Y. Shiota, N. Mizuochi, T. Shinjo, and Y. Suzuki, Large Voltage-Induced Magnetic Anisotropy Field Change in Ferrimagnetic FeGd, Appl. Phys. Express 8, 073007 (2015).

[38] W. Thomson, On the Electro-Dynamic Qualities of Metals:Effects of Magnetization on the Electric Conductivity of Nickel and of Iron, Proc. R. Soc. London 8, 546 (1856).

[39] Y. Shiota, S. Murakami, F. Bonell, T. Nozaki, T. Shinjo, and Y. Suzuki, Quantitative Evaluation of Voltage-Induced Magnetic Anisotropy Change by Magnetoresistance Measurement, Appl. Phys. Express 4, 043005 (2011).

[40] L. Gao, X. Jiang, S.-H. Yang, J. D. Burton, E. Y. Tsymbal, and S. S. P. Parkin, Bias Voltage Dependence of Tunneling Anisotropic Magnetoresistance in Magnetic Tunnel Junctions with $\mathrm{MgO}$ and $\mathrm{Al}_{2} \mathrm{O}_{3}$ Tunnel Barriers, Phys. Rev. Lett. 99, 226602 (2007).

[41] A. Matos-Abiague, M. Gmitra, and J. Fabian, Angular Dependence of the Tunneling Anisotropic Magnetoresistance in Magnetic Tunnel Junctions, Phys. Rev. B 80, 045312 (2009).

[42] N. Néel, S. Schröder, N. Ruppelt, P. Ferriani, J. Kröger, R. Berndt, and S. Heinze, Tunneling Anisotropic Magnetoresistance at the Single-Atom Limit, Phys. Rev. Lett. 110, 037202 (2013).

[43] C. Tiusan, J. Faure-Vincent, C. Bellouard, M. Hehn, E. Jouguelet, and A. Schuhl, Interfacial Resonance State Probed by Spin-Polarized Tunneling in Epitaxial $\mathrm{Fe} / \mathrm{MgO} / \mathrm{Fe}$ Tunnel Junctions, Phys. Rev. Lett. 93, 106602 (2004).

[44] E. Y. Tymbal, K. D. Belashchenko, J. P. Velev, S. S. Jaswal, M. van Schilfgaarde, I. I. Oleynik, and D. A. Stewart, Interface Effects in Spin-Dependent Tunneling, Prog. Mater. Sci. 52, 401 (2007).

[45] Y. Shiota, F. Bonell, S. Miwa, N. Mizuochi, T. Shinjo, and Y. Suzuki, Opposite Signs of Voltage-Induced Perpendicular Magnetic Anisotropy Change in CoFeBMgO Junctions with Different Underlayers, Appl. Phys. Lett. 103, 082410 (2013).

[46] W. Skowroński, T. Nozaki, D. D. Lam, Y. Shiota, K. Yakushiji, H. Kubota, A. Fukushima, S. Yuasa, and Y. Suzuki, Underlayer Material Influence on ElectricField Controlled Perpendicular Magnetic Anisotropy in 
CoFeB/MgO Magnetic Tunnel Junctions, Phys. Rev. B 91, 184410 (2015).

[47] T. Nozaki, A. Kozioł-Rachwał, W. Skowrónski, V. Zayets, Y. Shiota, S. Tamaru, H. Kubota, A. Fukushima, S. Yuasa, and Y. Suzuki, Large Voltage-Induced Changes in the Perpendicular Magnetic Anisotropy of an MgO-Based Tunnel Junction with an Ultrathin Fe Layer, Phys. Rev. Applied 5, 044006 (2016).

[48] P. V. Ong, N. Kioussis, D. Odkhuu, P. Khalili Amiri, K. L. Wang, and G. P. Carman, Giant Voltage Modulation of Magnetic Anisotropy in Strained Heavy Metal/Magnet/ Insulator Heterostructures, Phys. Rev. B 92, 020407(R) (2015).

[49] P. V. Ong, N. Kioussis, P. K. Amiri, and K. L. Wang, Electric-Field-Driven Magnetization Switching and Nonlinear Magnetoelasticity in $\mathrm{Au} / \mathrm{FeCo} / \mathrm{MgO}$ Heterostructures, Sci. Rep. 6, 29815 (2016).

[50] F. Bonell, Y. T. Takahashi, D. D. Lam, S. Yoshida, Y. Shiota, S. Miwa, T. Nakamura, and Y. Suzuki, Reversible Change in the Oxidation State and Magnetic Circular Dichroism of Fe Driven by an Electric Field at the $\mathrm{FeCo} / \mathrm{MgO}$ Interface, Appl. Phys. Lett. 102, 152401 (2013).

[51] K. Leistner, J. Wunderwald, N. Lange, S. Oswald, M. Richter, H. Zhang, L. Schultz, and S. Fähler, Electric-Field Control of Magnetism by Reversible Surface Reduction and Oxidation Reactions, Phys. Rev. B 87, 224411 (2013).

[52] C. Bi, Y. Liu, T. Newhouse-Illige, M. Xu, M. Rosales, J. W. Freeland, O. Mryasov, S. Zhang, S. G. E. te Velthuis, and
W. G. Wang, Reversible Control of Co Magnetism by Voltage-Induced Oxidation, Phys. Rev. Lett. 113, 267202 (2014).

[53] U. Bauer, L. Yao, A. J. Tan, P. Agrawal, S. Emori, H. L. Tuller, S. Dijken, and G.S.D. Beach, Magneto-Ionic Control of Interfacial Magnetism, Nat. Mater. 14, 174 (2015).

[54] S. Miwa, K. Matsuda, K. Tanaka, Y. Kotani, M. Goto, T. Nakamura, and Y. Suzuki, Voltage-Controlled Magnetic Anisotropy in FeMgO Tunnel Junctions Studied by X-Ray Absorption Spectroscopy, Appl. Phys. Lett. 107, 162402 (2015).

[55] T. Tsukahara, T. Kawabe, K. Shimose, T. Furuta, R. Miyakaze, K. Nawaoka, M. Goto, T. Nozaki, S. Yuasa, Y. Kotani, K. Toyoki, M. Suzuki, T. Nakamura, Y. Suzuki, and S. Miwa, Characterization of the Magnetic Moments of Ultrathin Fe Film in an External Electric Field via HighPrecision X-Ray Magnetic Circular Dichroism Spectroscopy, Jpn. J. Appl. Phys. 56, 060304 (2017).

[56] S. Miwa, M. Suzuki, M. Tsujikawa, K. Matsuda, T. Nozaki, K. Tanaka, T. Tsukahara, K. Nawaoka, M. Goto, Y. Kotani, et al., Voltage Controlled Interfacial Magnetism through Platinum Orbits, Nat. Commun. 8, 15848 (2017).

[57] F. Schleicher, U. Halisdemir, D. Lacour, M. Gallart, S. Boukan, G. Schmerber, V. Davesne, P. Panissod, D. Halley, H. Majad et al., Localized States in Advanced Dielectrics from the Vantage of Spin- and Symmetry-Polarized Tunnelling across MgO, Nat. Commun. 5, 4547 (2014). 